\title{
Application of Adaptive Thresholds in Robust Fault Detection of an Electro- Mechanical Single-Wheel Steering Actuator
}

\author{
Lok Man Ho* \\ *System Dynamics and Control, Robotic and Mechatronic Center, \\ German Aerospace Center (DLR),Weßling, D-82234, Germany (email: lok.ho@dlr.de)
}

\begin{abstract}
A fault detection scheme has been developed for an electromechanical steering actuator under closed-loop control. The closed-loop system is modeled as a $2^{\text {nd }}$ order system with bounded parameter uncertainties given by the performance specifications. The residual is generated using an observer-based method. A dynamic adaptive threshold is generated based on the inputs and the measured system outputs to account for the residual deviating from zero under fault-free conditions due to parameter uncertainties. In addition to this, the error of the controlled system output due to a disturbance is handled by the residual evaluation function. This method is tested on a simple simulation as well as experimental data.
\end{abstract}

Keywords: model-based fault detection, adaptive threshold, steer-by-wire, parameter uncertainty

\section{INTRODUCTION}

Automotive steer-by-wire (SBW) systems have been investigated extensively in order to exploit the additional degrees of control freedom for vehicle dynamics control to improve safety and drive assistance functions. However, the failure of a steering actuator can lead to severe vehicle instability (Niederkofler et al. (2011)), making it a safety critical system. A fault should therefore be detected in a timely fashion, to allow reactive measures, such as control reconfiguration, to be taken to restabilise the vehicle. Several studies have been conducted regarding fault detection of SBW systems. These include approaches viewing the actuator at the level of the electric motor (Thomsen \& Blanke (2006)), considering the steering system as a whole including the forces arising from the road-wheel interaction (Pisu et al. (2004)), or a combination the above (Gadda (2008)).

Handling the effects of unknown disturbances and model uncertainty is an important consideration in practical fault detection algorithms. In active approaches to robustness, the effects of disturbances and uncertainties are completely or approximately decoupled from the effects of faults on the residual using nullspace methods (Varga (2011)). Passive approaches to robust fault detection and isolation (FDI) calculate the effects of these uncertainties on the residual and consider this in the evaluation of the residual value to decide on the fault status of the system. One such approach, the use of adaptive thresholds, has been applied in literature to systems with bounded parametric uncertainties using a variety of methods. One method is the use of interval observers to produce a bound for the possible system states (Benothman et al. (2007), Puig et al. (2008)), while another involves the manipulation of state and error dynamic equations to calculate an upper bound on the residual (Pisu et al. (2004), Hashemi \& Pisu (2011)).

The main contribution of this paper is the extension of the method in (Hashemi \& Pisu (2011)) to deal directly with uncertainties in the physical model parameters. The steering actuator from the ROboMObil (Brembeck et al. (2011)) provides an application example. The ROboMObil is a prototype intelligent electric vehicle with four wheels that are independently driven and steered by electromechanical actuators integrated into "wheel robot" units (Figure 1). These are characterised by their ability to steer through a total angle range of approximately $125^{\circ}$, thus enabling sideway driving.

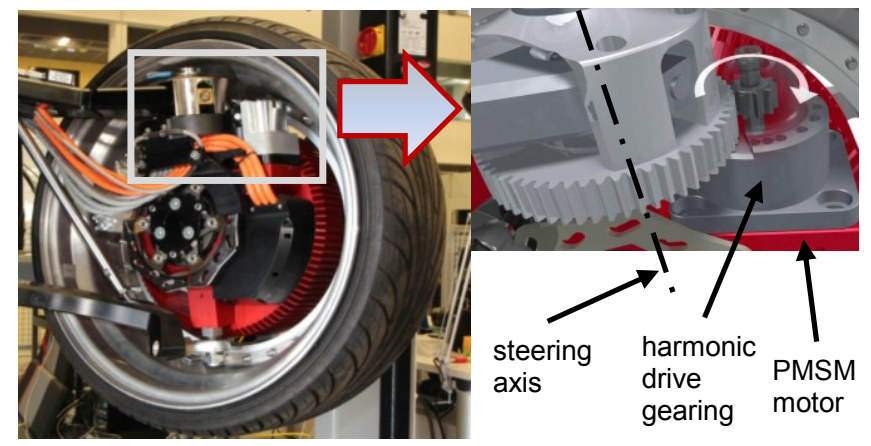

Figure 1: The electromechanical steering actuator integrated into the "wheel-robots"

The paper is organised as follows. Section 2 describes the model of the electromechanical steering actuator. Sections 3 and 4 describe the fault detection scheme and the calculation of the adaptive threshold. Application of the proposed methods to the steering actuator is presented in section 5, with numerical validation using simulations and experimental data in section 6 . This is followed by a closing discussion and description of future work in section 7.

\section{SYSTEM DESCRIPTION}

The steering actuator consists of a permanent magnet synchronous motor (PMSM) and a harmonic drive gear system. Its output shaft is connected to a spur gear which meshes with a larger spur gear rotationally fixed to the steering axis. Angular position sensors are mounted on the motor's rotor as well as on the steering axis. The local 
position controller in Figure 2 receives an angular position demand from the vehicle dynamics controller (VDC). The fault diagnosis module needs to detect critical failures in the vehicle, one of which is the failure of the steering actuator to track the demanded position, with only the demanded and the measured values of the steering position available.

\subsection{Block Diagram of the Steer-By-Wire Actuator}

The electromechanical actuator is controlled by a cascade architecture, with an inner angular velocity control loop and an outer angular position control loop (see Figure 2). The dynamics of the current control in the field-oriented controller (FOC) has a significantly lower time constant compared to the mechanical dynamics, therefore we will assume that the rate controller commands the motor current directly. Friction in the actuator is considered as part of torque disturbance $d$. The saturation for motor current is ignored in this analysis as it is rarely reached in normal operation. The rate limiter is activated regularly in normal operation, but it will not be considered in this analysis.

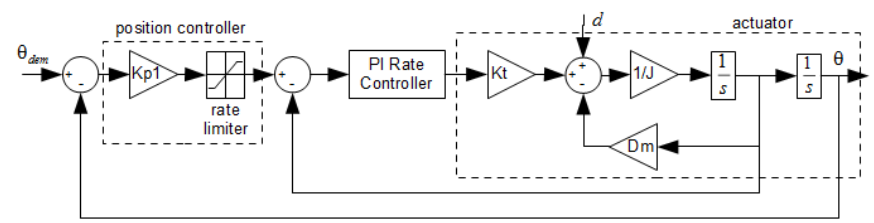

Figure 2: Block diagram representation of the controlled steering actuator

In the case where the rate limiter has not reached saturation, the transfer function from the demanded steering angle to the actual steering angle is given by:

$$
\frac{\theta(s)}{\theta_{d e m}(s)}=\frac{K_{p 1} K_{t}\left(K_{i}+K_{p} s\right)}{J s^{3}+\left(K_{t} K_{p}+D_{m}\right) s^{2}+K_{t}\left(K_{i}+K_{p 1} K\right) s+K_{t} K_{i} K_{p 1}}
$$

The parameters definitions and their values for the actuator under investigation are defined in Figure 2 and Table 1.

Table 1. Controlled actuator model parameters

\begin{tabular}{|l|l|l|}
\hline Parameter & Symbol & Value \\
\hline Total inertia ( at motor) & $J$ & $4 \mathrm{e}^{-5} \mathrm{kgm}^{2}$ \\
\hline Total damping (at motor) & $D_{m}$ & $5 \mathrm{e}^{-4} \mathrm{Nms} / \mathrm{rad}$ \\
\hline Motor torque constant & $K_{t}$ & $0.053 \mathrm{Nm} / \mathrm{A}$ \\
\hline Rate control P-gain & $K_{p}$ & 0.115 \\
\hline Rate control I-gain & $K_{i}$ & 3.34 \\
\hline Position control P-gain & $K_{p l}$ & 40 \\
\hline Motor rate limit & $\omega_{r l}$ & $366.5 \mathrm{rad} / \mathrm{s}$ \\
\hline
\end{tabular}

\subsection{Approximation with a Black-box Actuator Model}

The real actuators are affected by uncertain non-linear effects such as internal friction, as well as unknown external load torques arising mainly from forces at the tyre-road interface, which are dependent on vehicle dynamic states, complex tyre behaviour and environmental parameters (Rajamani (2006)). The controller is specifically designed to robustly address these phenomena, with possible measures such as gain scheduling, friction compensation and suppression of mechanical resonances through frequency shaping filters. For the design of the fault detector, we consider a simplified "black-box" view of the actuator under closed-loop position control, with bounded dynamic properties given by performance specifications. The method remains applicable when the closed-loop system model is approximately determined through system identification techniques. The aim of this work is to detect a deviation from this specified behaviour while minimising false triggers due to modelling uncertainties that are defined as acceptable.
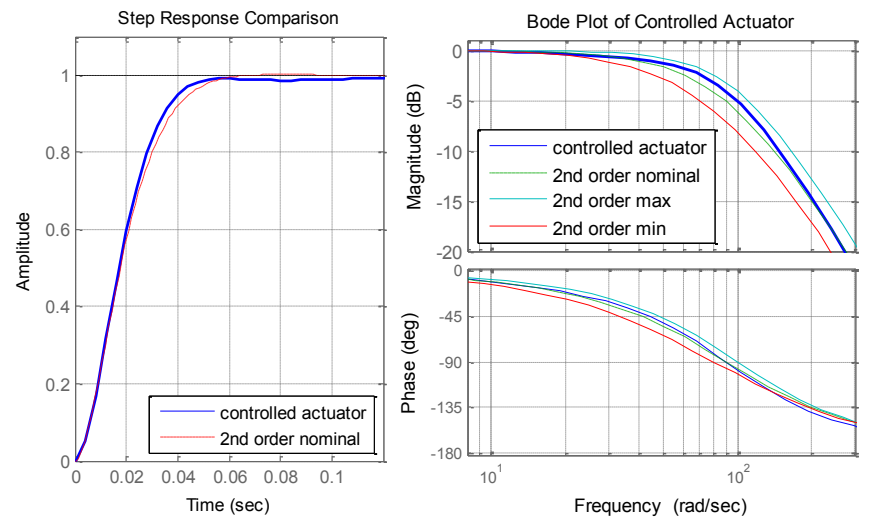

Figure 3: Left: Step responses of the modelled steering actuator and the $2^{\text {nd }}$ order approximation, Right: Bode plot of the controlled actuator compared to those given by the parameter bounds

From Figure 3, it can be seen that the frequency response of the actuator transfer function (1) is closely approximated by the second order filter transfer function with the following state space form.

$$
\begin{aligned}
\dot{x} & =\left[\begin{array}{cc}
-D & -K \\
1 & 0
\end{array}\right] x+\left[\begin{array}{l}
K \\
0
\end{array}\right] u \\
y & =\left[\begin{array}{ll}
0 & 1
\end{array}\right] x
\end{aligned}
$$

where $D=2 \zeta \omega_{n}, K=\omega_{n}^{2}$, with the parameter values $\omega_{n}=90 \mathrm{rad} / \mathrm{s}, \zeta=0.9$. The input is given by $u=\theta_{\text {dem }}$, and the output is $y=\theta$. Figure 3 also shows a comparison of the step responses. The frequency response of the controlled actuator is compared to those of the black-box model at the limits of the parameter bounds in the bode plot shown in Figure 3. The parameters have the ranges:

$$
\omega_{n} \in[75 \mathrm{rad} / \mathrm{s}, 105 \mathrm{rad} / \mathrm{s}], \zeta \in[0.8,1.0]
$$

We can see that the nominal frequency response of the closed-loop system lies within the tolerance band.

\section{FAULT DETECTION SCHEME}

The fault detection architecture is based on the generation of a residual utilising an observer based method. An adaptive threshold is calculated based on the inputs, system states and bounds on model uncertainties. The residual is compared to this dynamic threshold and the result is further processed before making a decision on the fault status. The general fault detection architecture is depicted in Figure 4. In this application, we have scalar inputs and outputs of $u=\theta_{\text {dem }}$ and $y=\theta$ respectively. 


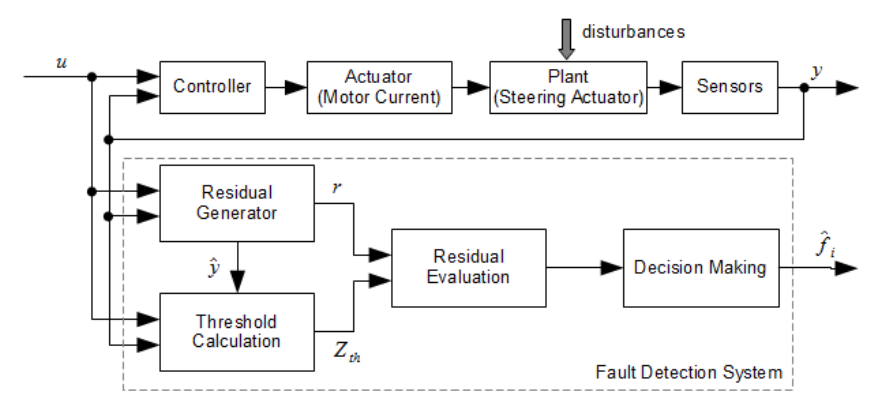

Figure 4: General fault detection architecture

The residual of the fault detector is generated by a conventional observer based scheme (Chen \& Patton (1999)). With the quad-tuple $\left\{A_{0}, B_{0}, C_{0}, 0\right\}$ with $A_{0} \in \mathbb{R}^{n \times n}, B_{0} \in$ $\mathbb{R}^{n \times m}$ and $C_{0} \in \mathbb{R}^{1 \times n}$ defining the nominal state space system, the fault detector is described by the equations:

$$
\begin{aligned}
& \dot{\hat{z}}=A_{0} \hat{z}+B_{0} u+L(y-\hat{y}) \\
& \hat{y}=C_{0} \hat{z} \\
& r=y-\hat{y}
\end{aligned}
$$

where $\hat{z}$ and $\hat{y}$ are the estimated states and outputs respectively, $r$ is the residual and $L$ is chosen such that $\left(A_{0}-L C_{0}\right)$ has all its eigenvalues in the open left half plane. In this case, $L$ is determined by the pole placement technique.

\section{ADAPTIVE THRESHOLD}

\subsection{Existing Method in Literature}

A method for the generation of adaptive thresholds to account for the variation of the residual from zero due to additive parametric uncertainties in an LTI system is proposed in (Hashemi \& Pisu (2011)). The system is first transformed into observer canonical form with an appropriate matrix $M$ and the transformation $z=M x$ :

$$
\begin{aligned}
& \dot{z}=\underbrace{\left[\begin{array}{ccccc}
-a_{n-1} & 1 & 0 & \ldots & 0 \\
-a_{n-2} & 0 & 1 & \ldots & 0 \\
\vdots & \vdots & \vdots & \ddots & \vdots \\
-a_{1} & 0 & 0 & \ldots & 1 \\
-a_{0} & 0 & 0 & \ldots & 0
\end{array}\right]}_{A_{0}} z+\underbrace{\left[\begin{array}{cccc}
b_{n-1,0} & \ldots & b_{n-1, \mathrm{~m}-1} \\
\vdots & \ldots & \vdots \\
\vdots & \vdots & \vdots \\
\vdots & \ldots & \vdots \\
b_{0,0} & \ldots & b_{0, \mathrm{~m}-1}
\end{array}\right]}_{B_{0}} \\
& y=\underbrace{\left[\begin{array}{lllll}
1 & 0 & \ldots & 0 & 0
\end{array}\right]}_{C_{0}} z
\end{aligned}
$$

The additive uncertainties in the parameters $a_{i}$ and $b_{i, j}$ grouped into the matrices $\Delta A_{0}$ and $\Delta B_{0}$ respectively. $f_{i}$ and $f_{o}$ are input and output fault vectors, with $B_{f i}$ and $B_{f o}$ the corresponding input matrices. Process and output noise are given by $w$ and $v$. We can then write the system equations as:

$$
\begin{aligned}
& \dot{z}=\left(A_{0}+\Delta A_{0}\right) z+\left(B_{0}+\Delta B_{0}\right) u+B_{f i} f_{i}+w \\
& y=C_{0} z+B_{f o} f_{o}+v
\end{aligned}
$$

With the observer defined by (3), and noting $\Delta A_{0} z=\Delta a y$, where $\Delta a=-\left[\Delta a_{n-1} \Delta a_{n-2} \cdots \Delta a_{0}\right]^{T}$ is the first column of $\Delta A_{0}$, the observer error is given in the noise and fault-free case by:

$$
\begin{aligned}
& e=z-\hat{z} \\
& \dot{e}=\left(A_{0}-L C_{0}\right) e+\Delta a y+\Delta B_{0} u \\
& r=y-\hat{y}=C_{0} e
\end{aligned}
$$

where $r$ is the residual and $e$ is the estimation error state. The use of the observer canonical form allows the measured $y$ to be used in the error equation instead of the unknown states of the real system $z$, assuming that there is no sensor noise or fault. The residual $r$ can be written as the time domain solution of the linear system with input $\Delta a y+\Delta B_{0} u$. With $\varepsilon_{0}(t)$ being the system response to the initial error state $e(0)$, we can write:

$$
\begin{aligned}
& r(t)=\varepsilon_{0}(t)+\int_{0}^{t} C_{0} e^{\left(A_{0}-L C_{0}\right)(t-\tau)} \Delta a y(\tau) d \tau+ \\
& \int_{0}^{t} C_{0} e^{\left(A_{0}-L C_{0}\right)(t-\tau)} \Delta B_{0} u(\tau) d \tau
\end{aligned}
$$

By considering each delta element of $\Delta a$ and $\Delta B_{0}$ separately and using the triangle inequality, this gives an error bound for $e_{1}(\mathrm{t})$ :

$$
\begin{aligned}
& |r(t)| \leq \\
& \varepsilon_{0}(t)+\sum_{i=1}^{n}\left|\int_{0}^{t} C_{0} e^{\left(A_{0}-L C_{0}\right)(t-\tau)} E_{i} \Delta a_{i-1} y(\tau) d \tau\right|+ \\
& \sum_{i=1}^{n} \sum_{j=0}^{m-1}\left|\int_{0}^{t} C_{0} e^{\left(A_{0}-L C_{0}\right)(t-\tau)} E_{i} \Delta b_{i-1, j} u_{j}(\tau) d \tau\right|
\end{aligned}
$$

The terms $\Delta a_{i-1}$ and $\Delta b_{i-1, j}$ are the uncertainties in the corresponding scalar parameters. $E_{i}$ is a column vector with all 0 s except a 1 as the $i$-th element. With the assumption that the scalar uncertainties $\Delta a_{i-1}$ and $\Delta b_{i-1, j}$ are constant, they can be moved outside of the integral. The integrals in (8) can then be evaluated as solutions to linear systems, with the output scaled by the uncertainty bounds (shown in (11) as $\delta_{a_{i-1}}$ and $\delta_{b_{i-1, j}}$ ) to provide the bounds on the error states. $Z_{t h}$ is the bounding value of $r$ and is used as the adaptive threshold value. Since $\left(A_{0}-L C_{0}\right)$ is stable through the choice of $L$, the term $\varepsilon_{0}(t)$ converges to zero at the time constant of the observer system and can be neglected after an initial period.

$$
\begin{aligned}
& \dot{\xi}_{i}=\left(A_{0}-L C_{0}\right) \xi_{i}+E_{i} y(t) \\
& \xi_{i, 1}=C_{0} \xi_{i} \\
& \dot{\psi}_{i j}=\left(A_{0}-L C_{0}\right) \psi_{i j}+E_{i} u_{j}(t) \\
& \psi_{i j, 1}=C_{0} \psi_{i j} \\
& Z_{t h}(t)=\varepsilon_{0}(t)+\sum_{i=1}^{n}\left(\delta_{a_{i-1}}\left|\xi_{i, 1}\right|+\sum_{j=0}^{m-1} \delta_{b_{i-1, j}}\left|\psi_{i j, 1}\right|\right)
\end{aligned}
$$

In the following subsections, an extension to this approach is proposed and the effects of faults on the threshold $Z_{t h}(t)$ is considered.

\subsection{Uncertainties in "Physical" Parameters}

It is likely that the parameters $a_{\mathrm{i}-1}$ and $b_{\mathrm{i}-1, \mathrm{j}}$ do not correspond directly to the vector of physical parameters $\mu$, due to the transformation into the observer canonical form. In fact, it is likely that a physical parameter such as $K_{t}$ will appear in several of the $a_{\mathrm{i}-1}$ and $b_{\mathrm{i}-1, \mathrm{j}}$ parameters. Instead of separately evaluating the error bounds due to the uncertainty in each $a_{\mathrm{i}-1}$ and $b_{\mathrm{i}-1, \mathrm{j}}$, it would be preferable to perform the separation according to $\mu$, since each parameter of $\mu$ must have the same 
value in all instances. It is unnecessarily conservative to evaluate the worst case of each instance of a parameter separately as it appears in different terms.

Considering $u$ and $y$ together as the input matrix to the observer and error dynamic systems, we get

$r(t)=\varepsilon_{0}(t)+\int_{0}^{t} C_{0} e^{\left(A_{0}-L C_{0}\right)(t-\tau)}\left[\begin{array}{ll}\Delta B_{0} & \Delta a\end{array}\right]\left[\begin{array}{l}u(\tau) \\ y(\tau)\end{array}\right] d \tau$

Assuming that all matrix elements of $A_{0}$ and $B_{0}$ are linear in $\mu$, we can write the elements of the uncertainty matrices as:

$$
\begin{aligned}
& \Delta a_{i}=\frac{\partial a_{i}}{\partial \mu_{1}} \Delta \mu_{1}+\cdots+\frac{\partial a_{i}}{\partial \mu_{n}} \Delta \mu_{p} \\
& \Delta b_{i, j}=\frac{\partial b_{i, j}}{\partial \mu_{1}} \Delta \mu_{1}+\cdots+\frac{\partial b_{i, j}}{\partial \mu_{n}} \Delta \mu_{p}
\end{aligned}
$$

The matrix $\left[\begin{array}{ll}\Delta B_{0} & \Delta a\end{array}\right]$ can be rewritten as:

$$
\left[\begin{array}{ll}
\Delta B_{0} & \Delta a
\end{array}\right]=\sum_{k=1}^{p} \Delta \mu_{k}\left[\begin{array}{ll}
\frac{\partial B_{0}}{\partial \mu_{k}} & \frac{\partial a}{\partial \mu_{k}}
\end{array}\right]
$$

With the same steps as previously performed, we get the following equation for the error bounds:

$$
\begin{aligned}
& |r(t)| \leq \varepsilon_{0}(t) \\
& +\sum_{k=1}^{p} \delta_{\mu_{k}}\left|\int_{0}^{t} C_{0} e^{\left(A_{0}-L C_{0}\right)(t-\tau)}\left[\begin{array}{ll}
\frac{\partial B_{0}}{\partial \mu_{k}} & \frac{\partial a}{\partial \mu_{k}}
\end{array}\right]\left[\begin{array}{l}
u(\tau) \\
y(\tau)
\end{array}\right] d \tau\right|
\end{aligned}
$$

where $\delta_{\mu_{k}}$ is the bounding value for $\Delta \mu_{k}$. The error-bound corresponding to each $\Delta \mu_{k}$ is then given by:

$$
\begin{aligned}
& \dot{\xi}_{\mu_{k}}=\left(A_{0}-L C_{0}\right) \xi_{\mu_{k}}+\left[\begin{array}{ll}
\frac{\partial B_{0}}{\partial \mu_{k}} & \frac{\partial a}{\partial \mu_{k}}
\end{array}\right]\left[\begin{array}{l}
u(t) \\
y(t)
\end{array}\right] \\
& \xi_{\mu_{k}, 1}=C_{0} \xi_{\mu_{k}} \\
& Z_{t h}(t)=\varepsilon_{0}(t)+\sum_{k=1}^{p} \delta_{\mu_{k}}\left|\xi_{\mu_{k}, 1}\right|
\end{aligned}
$$

In the cases where the elements of $A_{0}$ and $B_{0}$ are not linear in $\mu_{k}$, for small values of $\Delta \mu_{k}$, equations 0 and 0 are approximately valid for small uncertainties, as this represents a linearisation around the nominal values of $\mu_{k}$.

\subsection{Effects of faults on the residual threshold}

Here we reconsider the influence of faults on the residual threshold compared to their effects on the residual. As a simplifying assumption in the following analysis, that the real system is assumed to take the nominal parameter values.

\section{Threshold Generator}

For the response of the threshold generator to input and output faults, consider 0 and 0 . The presence of a fault only affects the term $y(t)$, so it can be deduced that the change in the threshold $Z_{t h}$ due to a fault is bounded by the inequality in (19). The inequality arises because of a need to employ the triangle inequality in the derivation, due to the existence of the absolute value operator in the expression in 0 .

$$
\begin{aligned}
& \dot{\phi}_{\mu_{k}}=\left(A_{0}-L C_{0}\right) \phi_{\mu_{k}}+\frac{\partial a}{\partial \mu_{k}} y_{f}(t) \\
& \Delta \xi_{\mu_{k}, 1}=C_{0} \phi_{\mu_{k}}
\end{aligned}
$$

$$
\Delta Z_{t h}(t) \leq \varepsilon_{0}(t)+\sum_{k=1}^{p} \delta_{\mu_{k}}\left|\Delta \xi_{\mu_{k}, 1}\right|
$$

$y_{f}(t)$ is the component of measured output $y$ due to the combined effects of $f_{i}$ and $f_{o}$. The system in (5) can be used to evaluate $y_{f}$. In transfer function form we can write:

$$
Y_{f}(s)=H_{y_{-} f i}(s) F_{i}(s)+B_{f o} F_{o}(s)
$$

In (18), each $\Delta \xi_{\mu_{k}, 1}$ is determined by a filter with $y_{f}$ as input. The transfer function representation is:

$$
\begin{aligned}
\Delta \xi_{\mu_{k}, 1}(s) & =H_{\mu_{k}}(s) Y_{f}(s) \\
& =H_{\mu_{k}}(s)\left(H_{y_{-} f i}(s) F_{i}(s)+B_{f o} F_{o}(s)\right)
\end{aligned}
$$

\section{Residual Generator}

The residual dynamics as generated by the observer and real plant is described by the following equations in the presence of input and output faults:

$$
\begin{aligned}
e & =z-\hat{z} \\
\dot{e} & =\left(A_{0}-L C_{0}\right) e+\left[\begin{array}{ll}
B_{f i} & -L B_{f o}
\end{array}\right]\left[\begin{array}{l}
f_{i} \\
f_{o}
\end{array}\right] \\
r & =y-\hat{y}=C_{0} e
\end{aligned}
$$

The above system can be written in transfer function form, where $R_{f}(s)$ is the component of the residual due to the faults, and $H_{r_{-} f i}(s)$ and $H_{r_{-} f o}(s)$ transform the input and output faults respectively into the residual:

$$
R_{f}(s)=H_{r_{-} f i}(s) F_{i}(s)+H_{r_{-} f o}(s) F_{o}(s)
$$

\section{Change in threshold compared to change in residual}

For this method of calculating adaptive thresholds to be valid, the change in the threshold $\Delta Z_{t h}(t)$ due to the $f_{i}$ and $f_{o}$ must be less than the change in the residual $r$ due to those same faults. Using the final value theorem, the evaluation of this condition at steady state is represented by the following for step inputs of $f_{i}$ and $f_{o}$ respectively:

$$
\begin{aligned}
& \sum_{k=1}^{p} \delta_{\mu_{k}} \lim _{s \rightarrow 0}\left|H_{y_{-} f i}(s) H_{\mu_{k}}(s)\right|<\lim _{s \rightarrow 0}\left|H_{r_{-} f i}(s)\right| \\
& B_{f o} \sum_{k=1}^{p} \delta_{\mu_{k}} \lim _{s \rightarrow 0}\left|H_{\mu_{k}}(s)\right|<\lim _{s \rightarrow 0}\left|H_{r_{-} f o}(s)\right|
\end{aligned}
$$

Without loss of generality, $B_{f o}$ is assumed to be a scalar mapping one output fault $f_{o}$ to one output $y$. The fulfilment of (24) and (25) depends on $H_{y_{-} f i}(s)$ having only poles in the open left half plane, and the parameter uncertainties $\delta_{\mu_{k}}$ being sufficiently small. Note that the transfer function $H_{\mu_{k}}(s)$ must be stable due to the design of the error dynamics matrix $\left(A_{0}-L C_{0}\right)$. In practice, numerical simulations of step responses to faults can be used to determine whether the selected $\delta_{\mu_{k}}$ result in a valid adaptive threshold generator.

5 APPLICATION TO THE STEERING ACTUATOR

5.1 Application to the $2^{\text {nd }}$ Order Filter Approximation 
For the derivation of the adaptive threshold, we approximate the controlled steering actuator model by the second order filter model as mentioned above, with the state space representation in (2). This is first transformed into the observer canonical form with the transformation $z=M x$ :

$$
\begin{aligned}
& \dot{z}=\left[\begin{array}{ll}
-D & 1 \\
-K & 0
\end{array}\right] z+\left[\begin{array}{l}
0 \\
K
\end{array}\right] u \\
& y=\left[\begin{array}{ll}
1 & 0
\end{array}\right] z
\end{aligned}
$$

The parameters $D$ and $K$ are uncertain, with uncertainties of $\Delta D$ and $\Delta K$ respectively. Application of 0 and (15) results in the following bound $e_{1}(\mathrm{t})$ :

$$
\begin{aligned}
& |r(t)| \leq \\
& \varepsilon_{0}(t)+\delta_{D}\left|\int_{0}^{t} C_{0} e^{\left(A_{0}-L C_{0}\right)(t-\tau)}\left[\begin{array}{ll}
0 & 1 \\
0 & 0
\end{array}\right]\left[\begin{array}{l}
u(\tau) \\
y(\tau)
\end{array}\right] d \tau\right|+ \\
& \delta_{K}\left|\int_{0}^{t} C_{0} e^{\left(A_{0}-L C_{0}\right)(t-\tau)}\left[\begin{array}{cc}
0 & 0 \\
1 & -1
\end{array}\right]\left[\begin{array}{l}
u(\tau) \\
y(\tau)
\end{array}\right] d \tau\right|
\end{aligned}
$$

These can be implemented as state space linear systems, with the error bound given by $Z_{t h}$.

$$
\begin{aligned}
& \dot{\xi}_{D}=\left(A_{0}-L C_{0}\right) \xi_{D}+\left[\begin{array}{ll}
0 & 1 \\
0 & 0
\end{array}\right]\left[\begin{array}{l}
u(t) \\
y(t)
\end{array}\right] \\
& \xi_{D, 1}=C_{0} \xi_{D} \\
& \dot{\xi}_{K}=\left(A_{0}-L C_{0}\right) \xi_{K}+\left[\begin{array}{cc}
0 & 0 \\
1 & -1
\end{array}\right]\left[\begin{array}{l}
u(t) \\
y(t)
\end{array}\right] \\
& \xi_{K, 1}=C_{0} \xi_{K} \\
& Z_{t h}(t)=\varepsilon_{0}(t)+\delta_{D}\left|\xi_{D, 1}\right|+\delta_{K}\left|\xi_{K, 1}\right|
\end{aligned}
$$

\subsection{Sensitivity of the Residual to Disturbance}

The approximation of the response of the controlled actuator with (1) and (2) is based on the assumption that no torque disturbance (represented by $d$ in Figure 2) is applied to the system. When this is not the case, then we must consider the error that may be generated by such disturbances. We assume that the magnitude of the $d$ is bounded by $\gamma$. Due to the integral control action, the response of the output $\theta$ to a step input $d$ decays to zero.

The transfer function of disturbance torque to residual $\frac{R(s)}{d(s)}$ can be described by a state space system with 5 states incorporating the controlled actuator and the estimator models. The frequency response of the integral of the residual due to a disturbance input, $G_{1}(s)=\frac{1}{s} \frac{R(s)}{d(s)}$, has a finite value of $\varphi$ at $\omega=0$ (i.e. also zero gradient). This is also the maximum value of the frequency response function, which can in turn be interpreted as the L2 gain of the system. Since the disturbance is bounded by $|d| \leq \gamma$, the definition of the L2 gain bounds the value of the integral of the residual with $\left|\int r(t) d t\right|<\varphi \gamma$. The integrator function is therefore chosen as the residual evaluation function.

This approach is combined with the adaptive threshold by integrating $r(t)-Z_{t h}(t)$, considering the positive and negative thresholds separately:

$$
\begin{aligned}
& g_{+}(t)=\int_{0}^{t} \beta_{+}(t)\left(r(\tau)-Z_{t h}(\tau)\right) d \tau \\
& g_{-}(t)=\int_{0}^{t} \beta_{-}(t)\left(-Z_{t h}(\tau)-r(\tau)\right) d \tau
\end{aligned}
$$

When either $g_{+}(t)$ or $g_{-}(t)$ exceeds their respective minimum values (over time) by more than $\varphi \gamma$, we can conclude that the residual deviation is more than that produced by a disturbance bounded by $|d| \leq \gamma$. As an extension to this approach, $\beta_{+}(t)$ and $\beta_{-}(t)$ are parameters that can be set greater than 1 when $r(t)$ is on the zero side of the positive and negative thresholds respectively. This increases the rate of "forgetting" the last violation of the threshold.

The advantage of this method is that it does not induce an extra insensitive band on the residual threshold, leading to improved detection for faults with lower magnitudes which generate small values at the residual. This is at the expense of a slight delay in the detection due to the integral action. By including a second detection condition of $|r(t)|>Z_{d}$, where $Z_{d}$ is the maximum residual value due to a step input of $\gamma$ at $d$, the detection time for larger faults can be improved.

\section{SIMULATION AND EXPERIMENTAL RESULTS}

\subsection{Validation on Steering Actuator Plant Model}
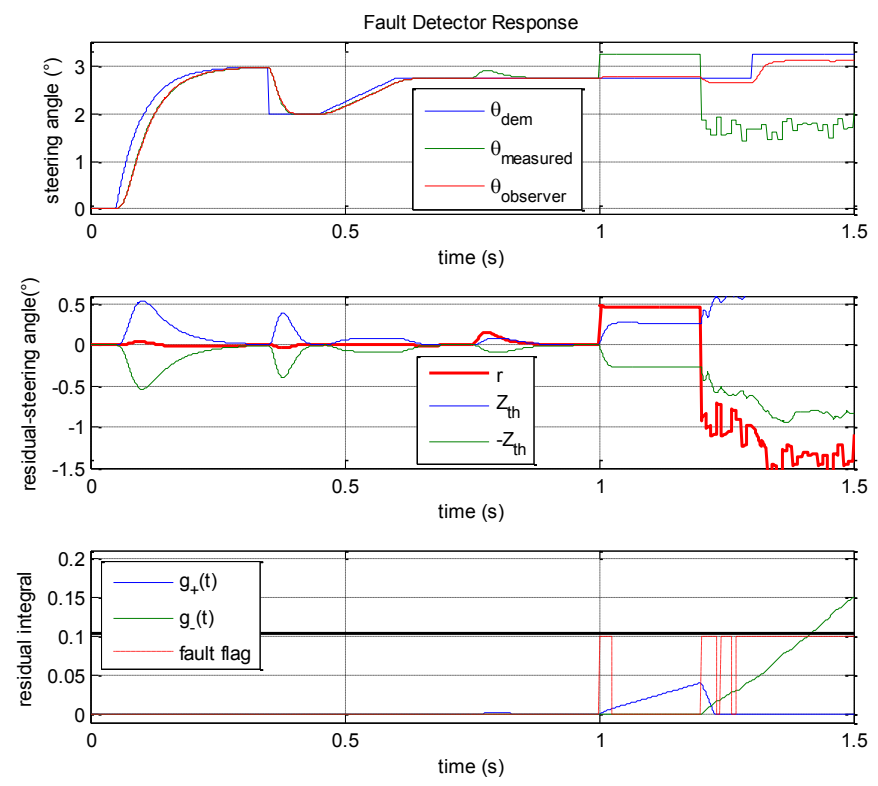

Figure 5: Adaptive threshold response to 2nd order model. The black line in the third plot shows the fault detection limit for $g_{+}$and $g_{\text {- }}$

The fault detection scheme was first tested on the steering actuator plant model, with the results shown in Figure 5. It can be seen that due to mismatch of the parameters between model and plant, the residual departs from zero when $\theta_{\text {dem }}$ changes, and then reduces back to zero again when the $\theta$ reaches $\theta_{\text {dem }}$. The adaptive threshold forms an envelope which encases the deviation of the residual from zero.

At $t=1 \mathrm{~s}$ a sensor offset fault is applied, followed by a sensor failure at $t=1.2 \mathrm{~s}$, where $\theta$ takes a random value near $\theta=2$ 
regardless of the value of $\theta_{d e m}$. In both of these cases, $|r|$ lies outside of the boundaries of the adaptive thresholds. The small magnitude of the sensor offset fault means that it would have been detected at around $1.5 \mathrm{~s}$ via the residual integral function (following the trend of $g_{+}(t)$ shown by the blue line). The sensor failure has a larger magnitude and triggers the detection condition $|r(t)|>Z_{d}$ immediately at $1.2 \mathrm{~s}$, and the integral evaluation function of $g_{-}(t)$ also exceeded the detection threshold at around $1.4 \mathrm{~s}$.

At $t=0.75 \mathrm{~s}$, a step torque disturbance of $100 \mathrm{Nm}$ is applied at the steering axis. Although the residual exceeds the threshold, the integral evaluation functions shown in the third plot remains below the threshold for a disturbance and thus no fault is detected, as desired.

\subsection{Validation on Steering Actuator Test Data}

The fault detection scheme with adaptive thresholds was also tested on data from the real actuator; see Figure 6 for the results. The data was taken from a test in which the ROboMObil was driven at approximately $10 \mathrm{~km} / \mathrm{h}$.
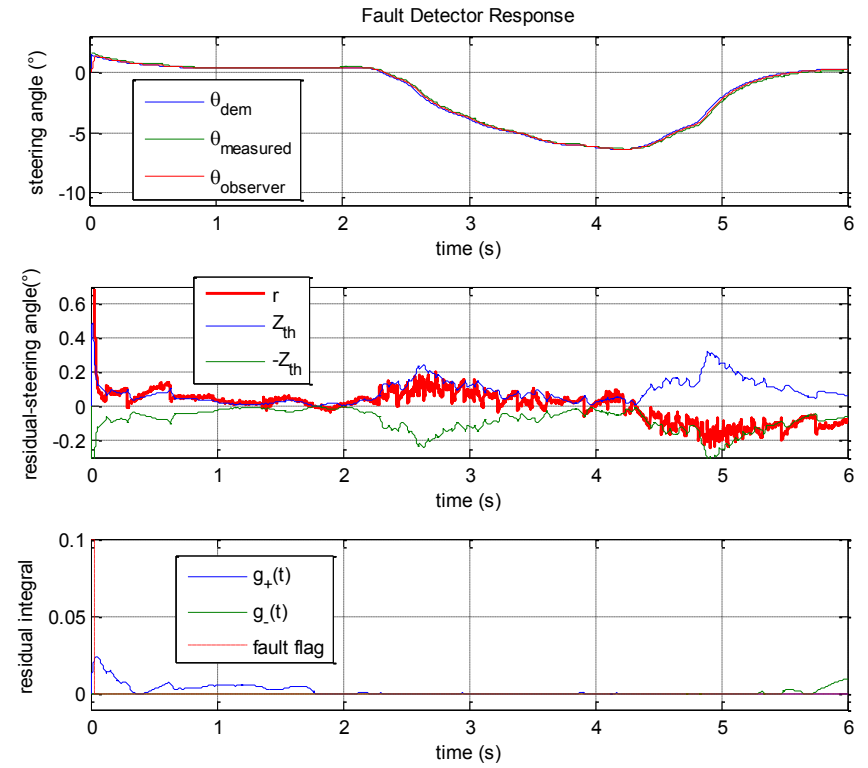

Figure 6: Threshold response to experimental test data

In this case, no fault was simulated. The residual is generally bounded by the adaptive threshold, whose deviation from zero can be seen to increase when actuator activity increases. In some cases, the residual does exceed the threshold, possibly due to nonlinear effects or unmodelled dynamics. In practice, this can be remedied by using an additional static threshold. The issue will be investigated further.

\section{CONCLUSION AND FUTURE WORK}

In this paper, a model-based fault detection method robust to parameter uncertainties for an actuator under closed-loop control was presented. This is achieved using a second-order representation of the system with parameter bounds for residual generation, together with adaptive residual thresholds. An extension to the methods in literature led to less conservative thresholds by considering uncertainties in physical parameters rather than in matrix elements. Future investigations will include the analysis of the effect of structural difference between the nominal model in (1) and the simplified second order model. Also to be considered will be the use of the observer output instead of the measured output to calculate the threshold in order to reduce its sensitivity to noise and faults, as well as handling of the nonlinearity introduced by the rate limiter.

\section{ACKNOWLEDGEMENTS}

The author would like to thank the DLR for providing the modelling and test data from the ROboMObil project, as well as Dr. Kaushik Mahata from the University of Newcastle, Australia for his support.

\section{REFERENCES}

Benothman, K., Maquin, D., Ragot, J. \& Benrejeb, M. (2007), 'Diagnosis of uncertain linear systems: an interval approach', International Journal of Sciences and Techniques of Automatic control \& computer engineering 1(2), 136-154. Brembeck, J., Ho, L., Schaub, A., Satzger, C., Tobolar, J., Bals, J. \& Hirzinger, G. (2011), Romo - the robotic electric vehicle, in '22nd IAVSD International Symposium on Dynamics of Vehicle on Roads and Tracks, 14-19 August, 2011'.

Chen, J. \& Patton, R. J. (1999), Robust model-based fault diagnosis for dynamic systems, Kluwer Academic Publishers, Norwell, MA, USA.

Gadda, C. D. (2008), Optimal fault-detection filter design for steer-by-wire vehicles, $\mathrm{PhD}$ thesis, Stanford University.

Hashemi, A. \& Pisu, P. (2011), Adaptive threshold-based fault detection and isolation for automotive electrical systems, in 'Intelligent Control and Automation (WCICA), 2011 9th World Congress on', pp. 1013-1018.

Niederkofler, H., Rojas, A. E. R. \& Dürnberger, J. (2011), Development of a single wheel steer-by-wire system: implementation aspects and failure handling, in '22nd IAVSD International Symposium on Dynamics of Vehicle on Roads and Tracks, 14-19 August, 2011', Institute of Automotive Engineering, Graz University of Technology, Graz, Styria, Austria.

Pisu, P., Silani, E. \& Rizzoni, G. (2004), Model-based sensor fault detection and isolation in a steer-by-wire system with parameter uncertainties, in 'FISITA '04'. 23-27 May 2004, Barcelona, Spain.

Puig, V., Quevedo, J., Escobet, T., Nejjari, F. \& de las Heras, S. (2008), 'Passive robust fault detection of dynamic processes using interval models', Control Systems Technology, IEEE Transactions on 16(5), 1083-1089.

Rajamani, R. (2006), Vehicle Dynamics and Control, Springer. ISBN 978-0-387-26396-0.

Thomsen, J. S. \& Blanke, M. (2006), Fault-tolerant actuator system for electrical steering of vehicles, in 'IEEE Industrial Electronics, IECON 2006 - 32nd Annual Conference on', Paris, France, pp. 3597-3602.

Varga, A. (2011), Synthesis of robust gain scheduling based fault detection filters for a class of parameter uncertain nonlinear systems, in 'Control and Automation (MED), 2011 19th Mediterranean Conference on’, pp. $467-472$. 